\title{
High-Q Free-standing Silicon Nitride Micordisk Vertically Coupled with On-chip Waveguide
}

\author{
Weiqiang Xie and Dries Van Thourhout \\ Photonics Research Group, Department of Information Technology (INTEC), Ghent University-IMEC, Gent B-9000, Belgium \\ weiqiang.xie@intec.ugent.be
}

\begin{abstract}
We designed and fabricated silicon nitride micordisk-waveguide vertical coupling devices processed at a low temperature of $270^{\circ}$. We experimentally demonstrate an intrinsic quality factor of $7.2 \times 10^{4}$ in the disk with only $15 \mu \mathrm{m}$ radius operating near $1310 \mathrm{~nm}$. OCIS codes: (130.0130) Integrated Optics; (140.3948) Microcavity devices.
\end{abstract}

\section{Introduction}

On-chip SiN resonators with small footprint are considered as critical building blocks in the variety of potential applications of integrated optics, photonic communications, and on-chip biosensing. However, because of its low optical index $(\sim 2.0)$, the fabrication of on-chip SiN resonator with a compact size and a high quality factor $(\mathrm{Q})$ still remains a challenge, and the most demonstrated high-Q $\mathrm{SiN}$ resonators such as ring and disk $[1,2]$ possess more than $50 \mu \mathrm{m}$ footprint which probably prevents their dense integration on chip.

In this work, we designed and fabricated SiN micordisk-waveguide vertical coupling devices using a home-built plasma enhanced chemical vapor deposition (PECVD) SiN platform developed at a low temperature of $270^{\circ}$. The experimental study on these devices reveals an intrinsic quality factor of $7.2 \times 10^{4}$ and an average transmission dip of $25 \mathrm{~dB}$ in the disk with only $15 \mu \mathrm{m}$ radius, operating near $1310 \mathrm{~nm}$. Furthermore, it is shown that the coupling strength between the disk resonator and the bus waveguide can be readily changed from undercoupling to overcoupling regime by tuning the parameters of the geometric configuration. Because of the small size of the disk and therefore the large free spectral range, these on-chip high-Q SiN resonators enable single mode operation within a wide wavelength spectrum.

\section{Design and Fabrication}

The geometric design of the SiN disk-waveguide vertical coupling device is shown in the Fig. 1 (a-c). The design starts with a silicon wafer with a $3 \mu \mathrm{m}$ thermal $\mathrm{SiO}_{2}$ box layer. The bus waveguide is buried in $\mathrm{SiO}_{2}$ matrix and the vertical coupling gap between the SiN disk and waveguide is determined by the thickness of the amorphous silicon (a-Si) pillar, as shown in Fig. 1(a-c). The top SiN disk aligned to the bus waveguide is suspended on the a-Si pillar with sufficient free hanging circumference to avoid the leakage of the fundamental transverse electric (TE) mode to the a-Si pillar. The offset here is defined as the horizontal distance between the edge of the disk and the center of the bus waveguide. As shown in cases of Fig. 1(a-c), the offset varies from $-300 \mathrm{~nm}$ to $+300 \mathrm{~nm}$ as the bus waveguide approaches towards the disk edge. By performing finite-difference time-domain simulations, we take the thickness of $\sim 400 \mathrm{~nm}$ for both waveguide and disk, and the vertical coupling gap is fixed at $\sim 300 \mathrm{~nm}$ for all devices. The offset, as one of the important coupling parameters [3], is changed from $-450 \mathrm{~nm}$ to $+450 \mathrm{~nm}$ by step of $150 \mathrm{~nm}$ on the contact mask design. The radius of the SiN disk is taken from $5 \mu \mathrm{m}$ to $15 \mu \mathrm{m}$ and the simulated Q for the uncoupled disk is shown in Fig. 1(d). Note that in Fig. 1(d) the modes for different radius disk are denoted as the form of TE $(m$, $n$ ) which represents different order TE modes of the disk according to their radial mode number $m$, and azimuthal number $n$. To illustrate a valuable comparison of the $\mathrm{Q}$ for various radius disk, we choose these fundamental modes in such way that the wavelength for all the mode is close to $1300 \mathrm{~nm}$. In Fig. 1(e) we also present the simulated field intensity for the fundamental mode of the disk with $5 \mu \mathrm{m}$ radius.

To fabricate the designed devices, we firstly deposit $\sim 400 \mathrm{~nm}$ PECVD SiN at $270^{\circ}$ on a wafer with a $3 \mu \mathrm{m} \mathrm{SiO}_{2}$ layer and then defined the bus waveguide by lab optical lithography using contact mask. Thanks to the high resolution contact lithography we developed in clean room and the improved reactive ion etching process, we can fabricate $630 \mathrm{~nm}$ wide single mode waveguide with vertical and smooth sidewalls working around $1310 \mathrm{~nm}$. The loss of the waveguide at $1310 \mathrm{~nm}$ was measured below $3 \mathrm{~dB} / \mathrm{cm}$, which implies the low loss in the PECVD SiN at $270^{\circ}$. After waveguide fabrication, a $1.2 \mu \mathrm{m}$ PECVD $\mathrm{SiO}_{2}$ deposition and the chemical mechanical planarization (CMP) processes are followed to achieve a planarized surface with the local roughness below $10 \mathrm{~nm}$ variation attributed to the excellent CMP process. The vertical coupling gap is grown by the PECVD a-Si layer, and the top SiN disk is aligned to the buried bus waveguide with the same process as the waveguide fabrication. To release the SiN disk, finally we utilize the alkaline based wet undercut etching to achieve a suspended SiN disk on the a-Si pillar. In Fig. 
2(a) and (b), we shows both the optical and scanning electron microscope (SEM) images of one of the fabricated devices with $15 \mu \mathrm{m}$ diameter disk. It is clear that the fabrication is excellent to achieve a free-standing disk and waveguide coupling device. The enlarged view of the coupling region between disk and waveguide is also shown in Fig. 2(c), from which the offset between disk and waveguide can be measured (for this device, the offset is about 200nm.).
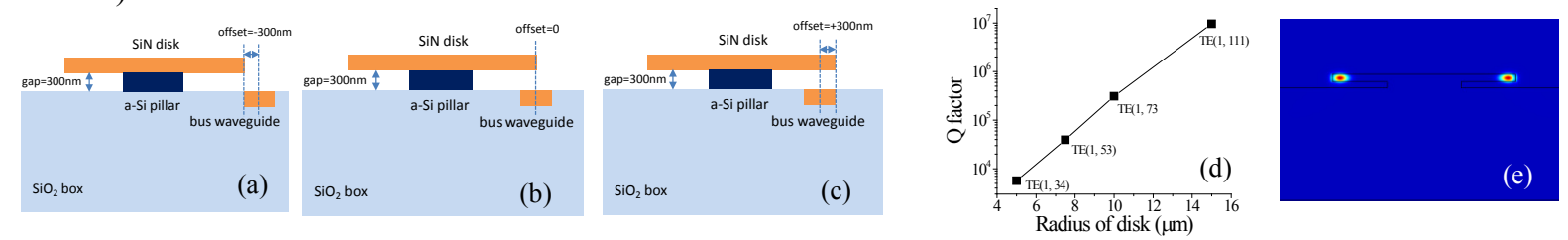

Fig. 1. (a-c) Cross-sectional schematics of SiN disk-waveguide vertical coupling configuration with different offset. (d) Simulated Q factors as the function of the radius of the disk. (e) Simulated field intensity profile of the fundamental mode for $5 \mu \mathrm{m}$ radius disk.
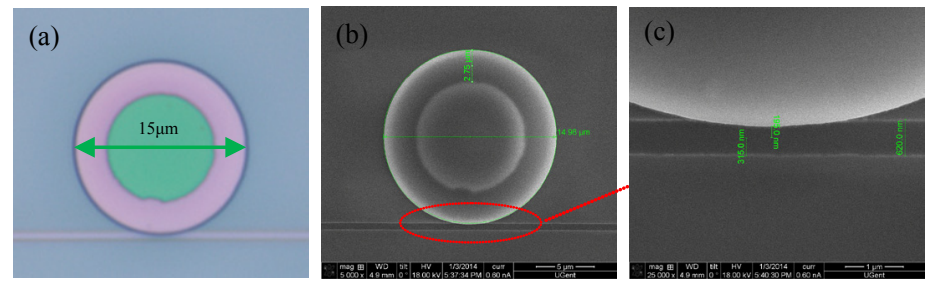

Fig. 2. (a) Optical and (b) SEM images of the top view of the device with $15 \mu \mathrm{m}$ diameter disk . (c) Enlarged view of the coupling region in (b).

\section{Characterization Results}

The disk-waveguide coupled devices were characterized by measuring the transmission spectra using a tunable laser with a wavelength range from 1260 to $1360 \mathrm{~nm}$ on a horizontal setup with lensed fibers coupled in and out to the cleaved facts of the bus waveguide. Fig. 3(a-d) shows the associated transmission of the disks with different radius. The average offset was measured $-200 \mathrm{~nm}$ for this group devices. It is obvious that when increasing the radius of disk the coupling between resonator and bus waveguide undergoes from undercoupling to critical coupling regime, which can be attributed to the increasing intrinsic $\mathrm{Q}$ as increasing the radius of the disk. Thus for higher intrinsic $\mathrm{Q}$, the weaker coupling strength is required to obtain the critical coupling. It is also clear that the transmission dip for the disks nearly working at critical coupling regime exhibits more that $20 \mathrm{~dB}$ transmission extinction. For the largest disk with the radius of $15 \mu \mathrm{m}$ even shows the average $30 \mathrm{~dB}$ transmission depth for all fundamental TE family modes over the whole spectrum, as shown in Fig. 3(d). As shown in Fig. 3(e) we extracted the coupled Q by a Lorentzian fitting and obtained Q of 36600 and 22000 for the typical transmission dips in Fig. 3(d) indicated with red circles. Considering the critical coupling circumstance, the intrinsic Q for these modes are two times of the measured Q [3], that is, the intrinsic $\mathrm{Q}$ of 72000 and 44000 for the two dips, respectively. For the smallest disk with radius of $5 \mu \mathrm{m}$, the measured Q still remains a considerable value. In addition, the coupling strength can be further tuned by changing the offset, and it is also found that for the larger disk the second TE modes can be selectively coupled out without exciting the fundamental modes by tuning the offset.
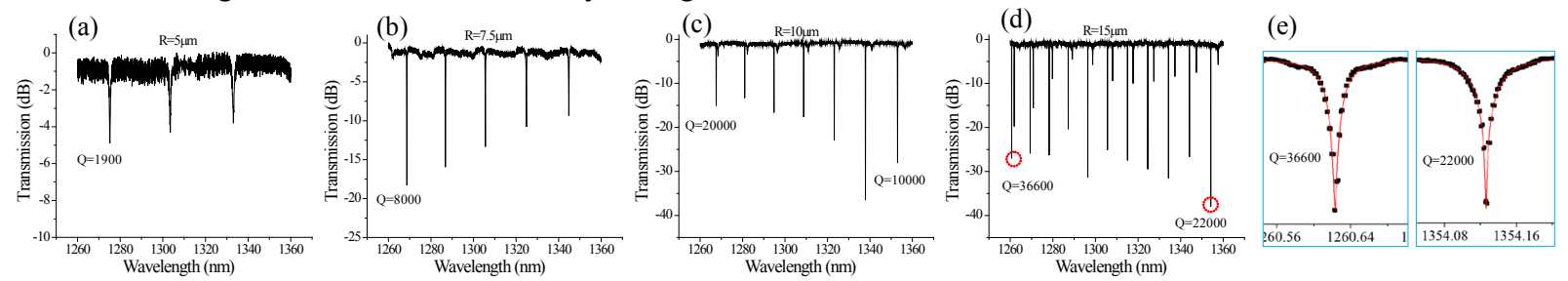

Fig. 3. (a-d) transmission spectra of the devices with different radius disks. (e) Fitted $Q$ for transmission tips in (d).

\section{Conclusion}

In this paper, we designed and fabricated SiN micordisk-waveguide vertical coupling devices with high-Q and compact footprints. These devices offer promising platform for the SiN photonic community.

\section{References}

[1] F. Ramiro-Manzano, et al., "A fully integrated high-Q Whispering-Gallery Wedge Resonator," Opt Express 20, 22934-22942 (2012).

[2] K. Luke, et al., "Overcoming $\mathrm{Si}_{3} \mathrm{~N}_{4}$ film stress limitations for high quality factor ring resonators," Opt Express 21, 22829-22833 (2013).

[3] F M. Ghulinyan, et al., "Oscillatory Vertical Coupling between a Whispering-Gallery Resonator and a Bus Waveguide," Phys Rev Lett 110 (2013). 